\title{
Buyang Huanwu decoction increases angiopoietin-1 expression and promotes angiogenesis and functional outcome after focal cerebral ischemia
}

\author{
Jian SHEN $^{1}$, Yu ZHU ${ }^{1}$, Hai YU ${ }^{1}$, Zuo-xu FAN ${ }^{1}$, Feng XIAO ${ }^{1}$, Pan WU ${ }^{1}$, Qi-hui ZHANG ${ }^{2}$, \\ Xiao-xing XIONG ${ }^{3}$, Jian-wei PAN ${ }^{\dagger 1}$, Ren-ya ZHAN ${ }^{\dagger 1}$ \\ ( ${ }^{1}$ Brain Medicine Center, the First Affiliated Hospital, School of Medicine, Zhejiang University, Hangzhou 310003, China) \\ ( ${ }^{2}$ Dongfang Hospital, Beijing University of Chinese Medicine, Beijing 100078, China) \\ ( ${ }^{3}$ Department of Anesthesiology, the First Affiliated Hospital, School of Medicine, Zhejiang University, Hangzhou 310003, China) \\ ${ }^{\dagger}$ E-mail: neurosurg_zju@yeah.net; neurovasword@gmail.com \\ Received June 18, 2013; Revision accepted Sept. 17, 2013; Crosschecked Feb. 4, 2014
}

\begin{abstract}
Buyang Huanwu decoction (BYHWD), a traditional Chinese herbal prescription, has been widely used clinically to treat stroke in China for hundreds of years; however, the mechanisms of this drug for stroke treatment are still unclear. This study aims to observe the cerebral angiogenesis effects of BYHWD on chronic brain injury after focal cerebral ischemia in rats and to explore its possible mechanisms. The ischemia was induced by occlusion of the right middle cerebral artery for $90 \mathrm{~min}$. BYHWD $(12.5$ and $25.0 \mathrm{~g} /(\mathrm{kg} \cdot \mathrm{d})$, equivalent to the dry weight of the raw materials) was orally administered twice a day beginning $2 \mathrm{~h}$ after surgery. BYHWD significantly attenuated the neurological dysfunction, infarct volume, and brain atrophy after ischemia. There was a significant increase in the microvessel density, as assessed by immunofluorescence CD31, and a significant increase in angiopoietin-1 (Ang-1) in the penumbra areas of the rats was shown by immunohistochemical staining and Western blotting. The results indicate that the neurorestorative effects of BYHWD are associated with angiogenesis and the enhancement of the expressions of Ang-1 on chronic brain injury after focal cerebral ischemia.
\end{abstract}

Key words: Buyang Huanwu decoction, Angiopoietin-1, Angiogenesis, Neurorestoration, Chronic brain injury doi: 10.1631 jzus.B1300166

Document code: A

CLC number: R743.3

\section{Introduction}

It is a well-known fact that stroke-evoked angiogenesis is likely to take place after brain ischemia, especially in the chronic phase (Wei et al., 2001; Bramlett and Dietrich, 2004). Angiogenesis, primarily in the ischemic boundary zone, enhances the supply of oxygen and nutrients to the affected tissue. Additionally, the generation of new blood vessels

\footnotetext{
Corresponding authors

* Project supported by the Plan of Zhejiang Scientific Research in Traditional Chinese Medicine (No. 2010ZZ007), China

(C) Zhejiang University and Springer-Verlag Berlin Heidelberg 2014
}

facilitates highly coupled neurorestorative processes, including neurogenesis and synaptogenesis, which in turn lead to improved functional recovery (Chen and Chopp, 2006; Beck and Plate, 2009). Studies from human and experimental stroke studies provide evidence for the therapeutic role of angiogenesis after focal cerebral ischemia.

Two angiogenic signal systems, the vascular endothelial growth factor (VEGF)/Flk and angiopoietins/ Tie-2 receptor, play key roles in the formation of new blood vessels. The VEGF/Flk system appears to be involved in blood vessel production in the acute stage of brain ischemia. The angiopoietins/Tie-2 
receptor system functions to maintain vascular networks in the later stages of brain ischemia (Suri et al., 1996). Angiopoietin-1 (Ang-1) signaling promotes angiogenesis and remodeling of blood vessels through its receptor tyrosine kinase Tie-2, which is contained in blood vessel endothelial cells. Transgenic overexpression of Ang-1 increases vascular stabilization (Suri et al., 1998; Liu et al., 2012), and Ang-1 and VEGF in combination induce a synergistic angiogenic effect and promote the formation of mature neovessels without the side effects on blood-brain barrier (BBB) permeability (Valable et al., 2005).

Buyang Huanwu decoction (BYHWD), a traditional Chinese herbal prescription, has been employed clinically to treat stroke in China for hundreds of years. A number of studies demonstrate that BYHWD improves the outcome of ischemic stroke in clinical trials (Hao et al., 2012). However, the mechanisms of this drug for stroke treatment are still unclear. Recent investigations showed its neuroprotective effects and mechanisms against cerebral ischaemia-reperfusion injury (Cai et al., 2007; Wang et al., 2011; Zhao et al., 2012). However, few studies have been performed to promote angiogenesis with BYHWD treatment, and the mechanisms of this drug for angiogenesis are unknown. In the present study, we investigate neurorestorative angiogenesis and the expressions of Ang-1 on chronic brain injury after focal cerebral ischemia.

\section{Materials and methods}

\subsection{Composition and preparation of BYHWD}

BYHWD was prepared as follows (Cai et al., 2007). BYHWD is composed of Radix Astragali, Radix Angelicae Sinensis, Radix Paeoniae Rubra, Rhizoma Chuanxiong, Semen Persicae, Flos Carthami, and Lumbricus, which were mixed at the ratio of 120:10:10:10:10:10:4.5 (dry weight). The decoction was made by boiling the mixture three times in distilled water at $100{ }^{\circ} \mathrm{C}$ for $30 \mathrm{~min}$. The drug solution was then vacuum-cooled and dried to form a powder, which was dissolved in distilled water at a final concentration of $2 \mathrm{~g} / \mathrm{ml}$ (equivalent to the dry weight of the raw materials). All materials were purchased from the East China Pharmaceutical Group Co., Ltd., Zhejiang Province, China.

\subsection{Animals and drug administration}

Adult male Sprague-Dawley rats, weighing 250$280 \mathrm{~g}$, were purchased from the Animals Laboratory Centre, Academy of Medical Sciences (Zhejiang, China) and all experiments were approved by the Animal Research Ethics Committee, School of Medicine, Zhejiang University, China. The rats were randomly divided into four groups: the sham-operated group (sham), the vehicle-treated middle cerebral artery occlusion (MCAO) group (vehicle), and MCAO groups treated with 12.5 and $25.0 \mathrm{~g} /(\mathrm{kg} \cdot \mathrm{d})$ BYHWD. The BYHWD was administrated orally twice a day starting $2 \mathrm{~h}$ after surgery. The vehicle and sham groups received an equal amount of distilled water for the same duration.

\subsection{Transient focal cerebra ischaemia-reperfusion model}

Focal cerebral ischemic rat models were induced by MCAO as previously reported (Cai et al., 2007; Xiong et al., 2013). In brief, the rats were anesthetized with $400 \mathrm{mg} / \mathrm{kg}$ of chloral hydrate i.p. The rats' rectal temperatures were maintained at $37{ }^{\circ} \mathrm{C}$ by placing the animals on a heating bed. The right carotid bifurcation was exposed, and the external carotid artery was coagulated distal to the bifurcation. A silicone-coated 4-0 suture was then inserted through the stump of the external cerebral artery and gently advanced $(17.0 \mathrm{~mm})$ to occlude the middle cerebral artery. After $90 \mathrm{~min}$ of occlusion, the filament was gently withdrawn, and the incision was closed. In the sham-operated rats, the common carotid was exposed without ligation.

\subsection{Neurological deficit}

The neurological behavior status was carefully evaluated once a week after surgery according to the method of Bederson et al. (1986). In brief, the neurological status was classified as follows: $0=$ no observable deficit; $1=$ forelimb flexion; $2=$ forelimb flexion plus decreased resistance to lateral push; $3=$ unidirectional circling; and $4=$ unidirectional circling plus decreased level of consciousness.

\subsection{Quantification of atrophy and infarct volume}

For evaluation of infarct volume, 2,3,5-triphenyl tetrazolium chloride (TTC; Sigma, St. Louis, MO, 
USA) staining was performed as previously described (Gu et al., 2013). Seven days after surgery, the rats were sacrificed under anesthesia, and their brains were immediately isolated and sliced rostrocaudally into serial 2-mm-thick slices. The sections of each brain were stained with $0.1 \%$ TTC solution and photographed. To correct for swelling due to cerebral edema, the infarct volume was normalized and expressed as a percentage of the ipsilateral hemispheric volume (Gu et al., 2013). For evaluation of the atrophy volume, the brains were removed and dehydrated four weeks after surgery, then cut into coronal slices of $2 \mathrm{~mm}$ thickness as described above. The atrophic area was calculated by subtracting out the area of the ipsilateral hemisphere from the area of the contralateral hemisphere and was expressed as a percentage of the ipsilateral hemispheric volume (Pang et al., 2001). The data were analyzed in a blinded manner using Image-Pro Plus 6.0 software (Media Cybernetics, Silver Spring, MD, USA).

\subsection{Immunofluorescence staining}

Frozen sections of $20-\mu \mathrm{m}$ thickness were prepared and blocked with a $10 \%$ normal serum blocking solution, with a species the same as the secondary antibody containing 3\% bovine serum albumin (BSA), $0.1 \%$ Triton $\mathrm{X}-100$, and $0.05 \%$ Tween-20 $2 \mathrm{~h}$ at room temperature in order to avoid unspecific staining. Then, the sections were incubated with primary rabbit anti-CD31 (1:100, Abcam, Cambridge, UK) overnight at $4{ }^{\circ} \mathrm{C}$, followed by goat anti-rabbit IgG conjugated by tetramethylrhodamine (TRITC) $(1: 200$, Jackson, PA, USA) for $2 \mathrm{~h}$ at $37^{\circ} \mathrm{C}$. Sections were covered with 4',6-diamidino-2-phenylindole (DAPI; Sigma, St. Louis, MO, USA) for $10 \mathrm{~min}$ at $30{ }^{\circ} \mathrm{C}$. The stained sections were examined with confocal laser scanning microscopy (Olympus, FV1000, Tokyo, Japan).

\subsection{Immunohistochemical staining}

Sections $(10-\mu \mathrm{m})$ were dewaxed and rehydrated using $100 \%, 95 \%$, and $70 \%$ of graded alcohol for $5 \mathrm{~min}$. The sections were then incubated for $2 \mathrm{~h}$ with $5 \%$ goat serum to block nonspecific immune reactions after the microwave antigen retrieval procedure (citrate buffer, $\mathrm{pH}$ 6.0). Anti-Ang-1 (1:50, Santa Cruz, CA, USA) was used as the primary antibody and incubated overnight at $4{ }^{\circ} \mathrm{C}$, followed by incubation with rabbit anti-goat $\operatorname{IgG}$ conjugated by horseradish peroxidase (HRP) (1:300, Cell Signaling, MA,
USA). Slides were then developed using a DAB (3,3'-diaminobenzidine) chromogen kit. The positive areas were measured using five randomly chosen fields. The intensity of positive staining in tissue sections was analyzed using the Image-Pro Plus 6.0 software (Media Cybernetics, Silver Spring, MD, USA) and SigmaPlot 4.0 (SPSS, Point Richmond, CA, USA).

\subsection{Measurement of the proteins in rat ischemic cortex by Western blotting}

The ischemic penumbra was homogenized in a radio-immunoprecipitation assay (RIPA) lysis buffer in the presence of a protease inhibitor. The supernatant was collected after the tissue was centrifuged at $12000 \times \mathrm{g}$ for $15 \mathrm{~min}$ at $4{ }^{\circ} \mathrm{C}$. The bicinchoninic acid protein assay (BCA; KeyGEN, Nanjing, China) was used to quantify the protein. In each lane, $40 \mu \mathrm{g}$ of sample was loaded to a sodium dodecyl sulfate polyacrylamide gel electrophoresis by using $12 \%$ Ready Gel. Proteins were transferred into polyvinylidene fluoride membranes for $90 \mathrm{~min}$. The membrane was blocked with $5 \%$ nonfat dry milk in phosphate buffered saline (PBS) containing $0.05 \%$ Tween- 20 for $2 \mathrm{~h}$ and incubated with primary goat anti-Ang-1 (1:200, Santa Cruz, CA, USA) or rabbit anti-GAPDH (glyceraldehyde 3-phosphate dehydrogenase; 1:1000, Abcam, Cambridge, UK) overnight at $4{ }^{\circ} \mathrm{C}$, and then followed by $2 \mathrm{~h}$ of incubation with rabbit anti-goat or goat anti-rabbit IgG conjugated by HRP (1:3000, Cell Signaling, MA, USA). Then, the membranes were developed using an enhanced chemiluminescence ECL reagent (Millipore, MA, USA). The image was scanned, and band intensity was quantified using the Image-Pro Plus 6.0 software.

\subsection{Statistical analysis}

Statistical data were represented as mean \pm standard deviation (SD). Statistical analysis was performed by one-way analysis of variance (ANOVA) followed by a Student's $t$-test. A difference with $P<0.05$ was considered statistically significant.

\section{Results}

\subsection{BYHWD reduced the infarct and atrophy volumes}

To investigate the protection of BYHWD against the local cerebral ischemia, this study assessed the 
infarct volume of the injured cerebrum. The obvious infarct difference was indicated at the end of one week (Fig. 1a). As is shown in Table 1, after being treated with BYHWD for one week, the infarct volume was reduced from $(27.27 \pm 2.28) \%$ (vehicle-treated group) to $(20.17 \pm 2.17) \%(12.5 \mathrm{~g} /(\mathrm{kg} \cdot \mathrm{d}), P<0.01)$ and $(15.02 \pm 1.74) \%(25.0 \mathrm{~g} /(\mathrm{kg} \cdot \mathrm{d}), P<0.01)$, respectively. To evaluate the degree of atrophy in the injured cerebrum, this study assessed the atrophy volume from two to four weeks, and there was a significantly decreased atrophy volume at the end of four weeks (Fig. 1b). As is shown in Table 1, after treatment with BYHWD, the atrophy volume was reduced from $(11.16 \pm 1.35) \%$ (vehicle-treated group) to $(9.77 \pm 1.05) \%$ $(12.5 \mathrm{~g} /(\mathrm{kg} \cdot \mathrm{d}), P<0.01)$ and $(7.50 \pm 1.53) \%(25.0 \mathrm{~g} /(\mathrm{kg} \cdot \mathrm{d})$, $P<0.01$ ), respectively.

(a)

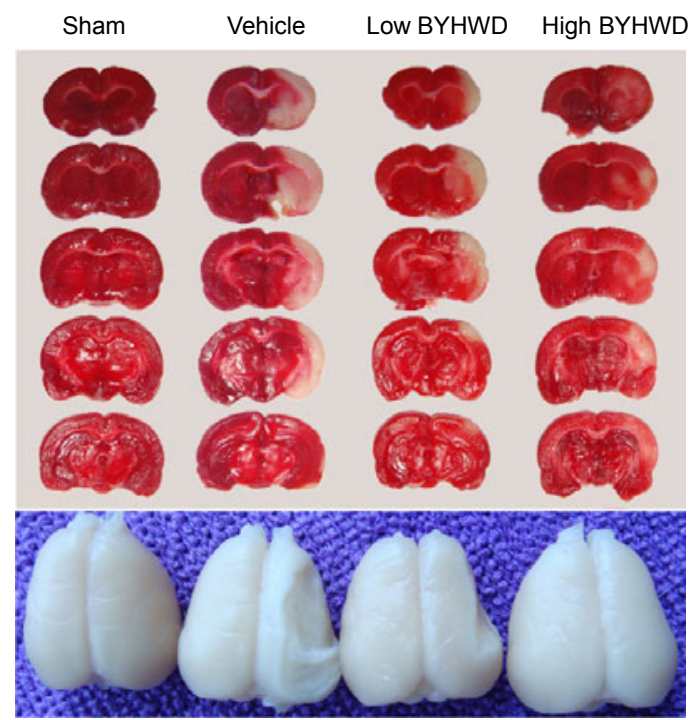

Fig. 1 Protective effect of BYHWD on infarct and atrophy volumes

(a) Representative TTC-stained coronal sections for each group one week after MCAO; (b) The atrophy percentage of each group four weeks after MCAO. Low BYHWD: $12.5 \mathrm{~g} /(\mathrm{kg} \cdot \mathrm{d})$; High BYHWD: $25.0 \mathrm{~g} /(\mathrm{kg} \cdot \mathrm{d})$

\subsection{BYHWD reduced neurological deficit}

To evaluate the neurological function, Bederson's score was used as the criteria for evaluation of the neurological deficit grading system which was carried out after MCAO. As is shown in Table 1, after treatment with BYHWD for one week, the neurological deficit improved from $2.2 \pm 0.63$ to $1.40 \pm 0.70(12.5 \mathrm{~g} /(\mathrm{kg} \cdot \mathrm{d})$, $P<0.05)$ and $1.10 \pm 0.74(25.0 \mathrm{~g} /(\mathrm{kg} \cdot \mathrm{d}), P<0.01)$. After two weeks, the deficit improved from $2.30 \pm 0.67$ to $1.30 \pm 0.67 \quad(P<0.01)$ and $0.90 \pm 0.73(P<0.01)$, and after three weeks, it improved from $2.00 \pm 0.47$ to $1.00 \pm 0.67(P<0.01)$ and $0.80 \pm 0.63(P<0.01)$. After four weeks of treatment, the deficit improved from $1.80 \pm 0.63$ to $0.80 \pm 0.63 \quad(P<0.01)$ and $0.50 \pm 0.53$ $(P<0.01)$, respectively. These results are in agreement with the results of the infarct volume variation.

\subsection{BYHWD increased microvessel density (MVD)}

The MVD, assessed by immunoreactive CD31, was performed as follows (Weidner, 2008). The immunostained sections were scanned at low magnification $(40 \times)$ and the area with the highest density of distinctly highlighted microvessels ("hot spot") was selected. The MVD was then determined in the hot spot by counting all vessels at a total magnification of $200 \times$. A score of 1 was given for each individual CD31-positive microvessel or single endothelial cell if they were clearly separated from the adjacent microvessels. Any vessel, even if incomplete, projecting into the field of view was scored. Ischemia-induced angiogenesis gradually increased in the penumbra area from two to four weeks, and BYHWD treatment further increased angiogenesis at four weeks after MCAO. As is shown in Fig. 2, the MVD was evidently increased in the vehicle-treated group from $17.73 \pm 5.98$ to $34.80 \pm 5.76(P<0.01)$, when compared with the sham-operated group at the end of four weeks. In the treatment groups with BYHWD, MVD markedly

Table 1 Effects of BYHWD on neurological deficit, infarct and atrophy volumes after focal cerebral ischemia

\begin{tabular}{|c|c|c|c|c|c|c|}
\hline \multirow{2}{*}{ Group } & \multicolumn{4}{|c|}{ Neurological deficit } & \multirow{2}{*}{$\frac{\text { Infarct volume }(\%)}{1 \text { week }}$} & \multirow{2}{*}{$\frac{\text { Atrophy volume }(\%)}{4 \text { weeks }}$} \\
\hline & 1 week & 2 weeks & 3 weeks & 4 weeks & & \\
\hline Sham & $0.00 \pm 0.00$ & $0.00 \pm 0.00$ & $0.00 \pm 0.00$ & $0.00 \pm 0.00$ & $0.00 \pm 0.00$ & $0.00 \pm 0.00$ \\
\hline Vehicle & $2.20 \pm 0.63$ & $2.30 \pm 0.67$ & $2.00 \pm 0.47$ & $1.80 \pm 0.63$ & $27.27 \pm 2.28$ & $11.16 \pm 1.35$ \\
\hline Low BYHWD & $1.40 \pm 0.70^{*}$ & $1.30 \pm 0.67^{* *}$ & $1.00 \pm 0.67^{* *}$ & $0.80 \pm 0.63^{* *}$ & $20.17 \pm 2.17^{* *}$ & $9.77 \pm 1.05^{* *}$ \\
\hline High BYHWD & $1.10 \pm 0.74^{* *}$ & $0.90 \pm 0.73^{* *}$ & $0.80 \pm 0.63^{* *}$ & $0.50 \pm 0.53^{* *}$ & $15.02 \pm 1.74^{* *}$ & $7.50 \pm 1.53^{* *}$ \\
\hline
\end{tabular}

Low BYHWD: $12.5 \mathrm{~g} /(\mathrm{kg} \cdot \mathrm{d})$ group; High BYHWD: $25.0 \mathrm{~g} /(\mathrm{kg} \cdot \mathrm{d})$ group. Data are expressed as mean $\pm \mathrm{SD}$ ( $n=10$ rats for neurological deficit, $n=10$ rats for infarct volume, and $n=6$ rats for atrophy volume). ${ }^{*} P<0.05$ or ${ }^{* *} P<0.01$ vs. the vehicle group 
increased to $51.00 \pm 11.39(12.5 \mathrm{~g} /(\mathrm{kg} \cdot \mathrm{d}), P<0.01)$ and $77.00 \pm 11.27(25.0 \mathrm{~g} /(\mathrm{kg} \cdot \mathrm{d}), P<0.01)$, respectively, in comparison to the vehicle-treated group.

\subsection{BYHWD increased protein expression of Ang-1 by immunohistochemical staining}

Our data demonstrated that the stroke-induced ischemic brain simultaneously and gradually increased the expression of Ang-1 beginning two weeks after $\mathrm{MCAO}$, and treatment with BYHWD further increased and maintained the expression of Ang-1 from three weeks after MCAO. As is shown in Fig. 3, there was little Ang-1 positive staining in the sham-operated

(a)

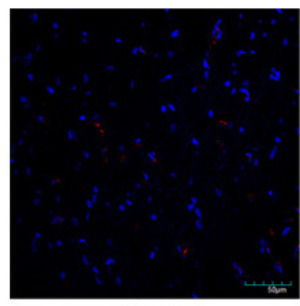

Sham

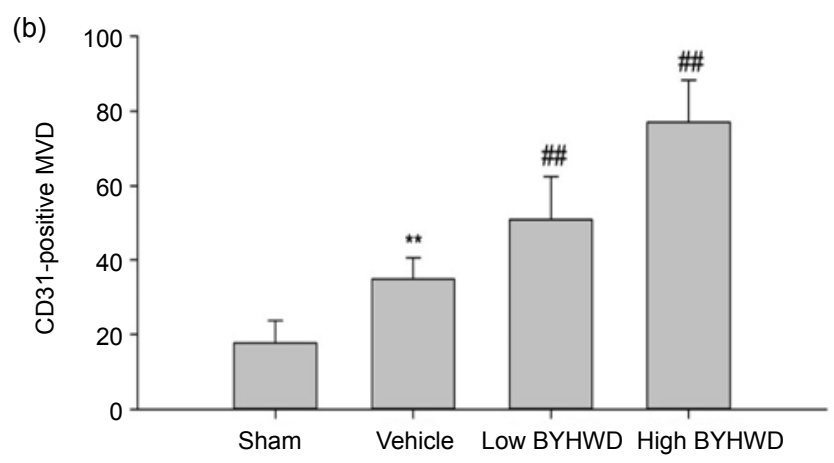

group. After ischemia at four weeks in the penumbra area, the positive Ang-1 staining remarkably increased in the vehicle-treated group (7022.30 $\pm 2549.58, P<0.01)$, when compared with the sham-operated group. In the BYHWD treatment groups, there was an evident increase in the positive Ang-1 staining to $11204.00 \pm$ $5898.38(12.5 \mathrm{~g} /(\mathrm{kg} \cdot \mathrm{d}), P<0.01)$ and $16179.00 \pm 6402$ $(25.0 \mathrm{~g} /(\mathrm{kg} \cdot \mathrm{d}), P<0.01)$, when compared with the vehicle-treated group. Furthermore, in the BYHWD treatment groups, Ang-1 protein was expressed not only in nerve tissue of the penumbra area but also in the brain microvascular endothelial cells constructing the vascular structures.

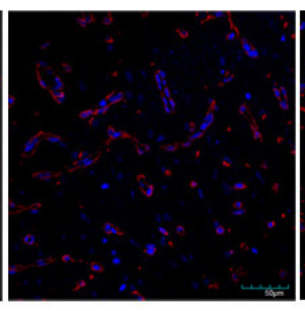

Low BYHWD

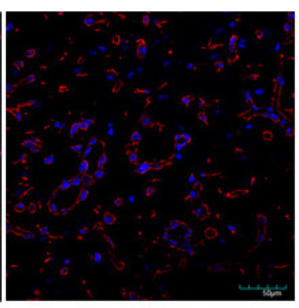

High BYHWD

Fig. 2 Microvessel density (MVD) in infarct area four weeks after MCAO for each group (200 $\times$ magnification) (a) CD31 and DAPI immunofluorescence stainings for each group four weeks after MCAO. (b) MVD was markedly increased by BYHWD treatment. Data are expressed as mean \pm SD of 6 rats per group. ${ }^{* *} P<0.01$ vs. the sham group; ${ }^{\#} P<0.01$ vs. the vehicle group. Low BYHWD: $12.5 \mathrm{~g} /(\mathrm{kg} \cdot \mathrm{d})$; High BYHWD: $25.0 \mathrm{~g} /(\mathrm{kg} \cdot \mathrm{d})$

(a)
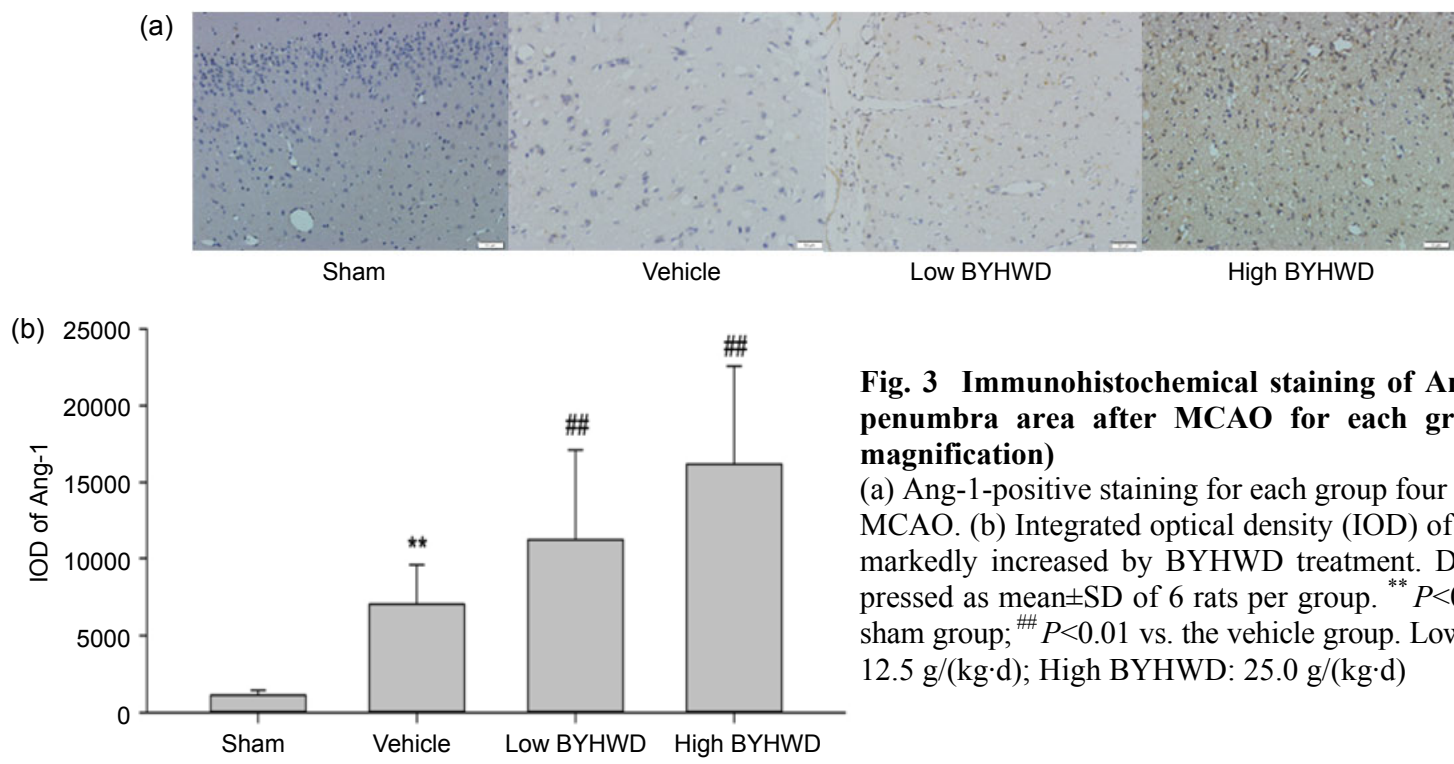

Fig. 3 Immunohistochemical staining of Ang-1 in the penumbra area after MCAO for each group (200× magnification)

(a) Ang-1-positive staining for each group four weeks after MCAO. (b) Integrated optical density (IOD) of Ang-1 was markedly increased by BYHWD treatment. Data are expressed as mean $\pm \mathrm{SD}$ of 6 rats per group. ${ }^{* *} P<0.01$ vs. the sham group; ${ }^{\#} P<0.01$ vs. the vehicle group. Low BYHWD: $12.5 \mathrm{~g} /(\mathrm{kg} \cdot \mathrm{d})$; High BYHWD: $25.0 \mathrm{~g} /(\mathrm{kg} \cdot \mathrm{d})$ 


\subsection{BYHWD increased protein expression of Ang-1 by Western blotting}

To further investigate the difference of the protein expression of Ang-1 in the penumbra area at four weeks, we utilized Western blotting. As is shown in Fig. 4, the level of Ang-1 protein in the vehicletreated group $(0.096 \pm 0.108, P<0.05)$ was higher than that in the sham-operated group, and after treatment with BYHWD for four weeks, the level of Ang-1 protein was dramatically increased to $0.134 \pm 0.014$ $(12.5 \mathrm{~g} /(\mathrm{kg} \cdot \mathrm{d}), P<0.01)$ and $0.163 \pm 0.010(25.0 \mathrm{~g} /(\mathrm{kg} \cdot \mathrm{d})$, $P<0.01)$, when compared with the vehicle-treated group. These results are in agreement with the results of the immunohistochemical staining.

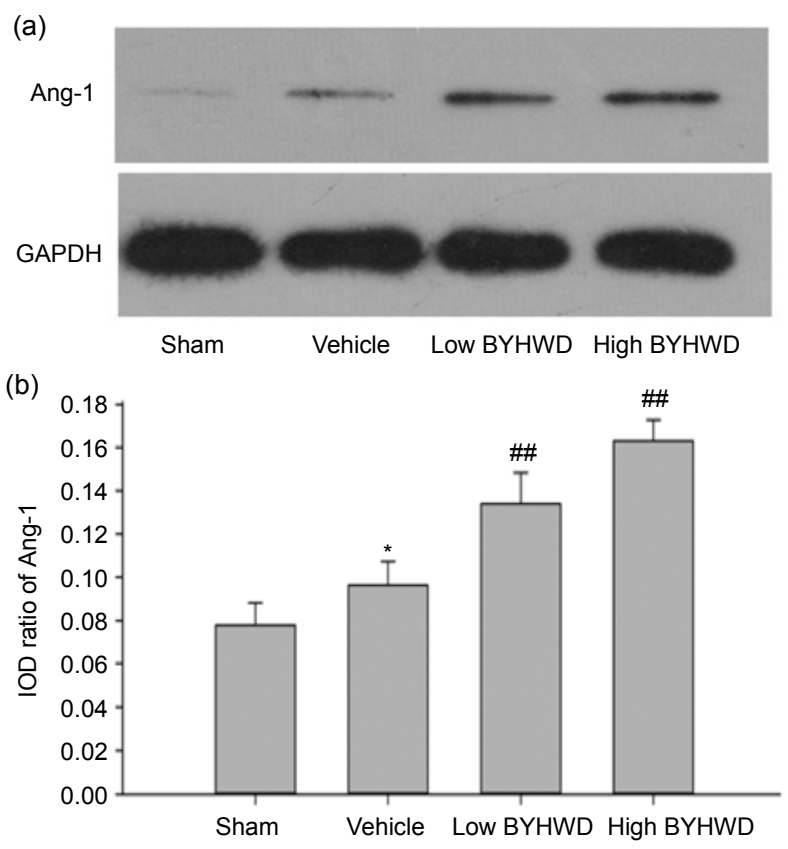

Fig. 4 Western blotting analysis of Ang-1 in the penumbra area after MCAO for each group

(a) Ang-1 Western blotting for each group four weeks after MCAO. (b) Integrated optical density (IOD) ratio of Ang-1 was markedly increased by BYHWD treatment. Data are expressed as mean \pm SD of 6 rats per group. ${ }^{*} P<0.05$ vs. the sham group, ${ }^{\#} P<0.01$ vs. the vehicle group. Low BYHWD: $12.5 \mathrm{~g} /(\mathrm{kg} \cdot \mathrm{d}) ;$ High BYHWD: $25.0 \mathrm{~g} /(\mathrm{kg} \cdot \mathrm{d})$

\section{Discussion}

For several decades, most attempts to treat stroke focused on neuroprotection (Fan and Yang, 2007).
However, only one Food and Drug Administration (FDA)-approved drug for the treatment of clinical ischemic stroke, a recombinant tissue plasminogen activator, shows therapeutic effects on the restoration of blood flow when given very early in carefully selected patients (Weintraub, 2006). Recent research has suggested that an alternative approach for restoring blood flow is to promote angiogenesis in regions surrounding the ischemic brain beyond the hyperacute phase of the stroke. The time window for therapies improving stroke recovery, so called neurorestorative therapies, is likely to be far longer than that for acute neuroprotection (Chen and Chopp, 2006; Beck and Plate, 2009). Neurorestorative events include neurogenesis, synaptic plasticity, and angiogenesis, for which the generation of new blood vessels as a neurorestorative event in the chronic stage after stroke, could be interpreted as a natural defense mechanism helping to restore the supply of nutrients to the affected brain tissue. Additionally, angiogenic vessels facilitate highly coupled neurorestorative processes, including neurogenesis and synaptogenesis, which in turn lead to improved functional recovery (Beck and Plate, 2009). These studies suggest that targeting brain vascular remodeling for drug discovery is necessary for the treatment of stroke and ischemia disease.

Many investigations showed the neuroprotective effects of BYHWD against cerebral ischaemiareperfusion injury (Cai et al., 2007; Wang et al., 2011; Zhao et al., 2012). The neuroprotection of BYHWD may involve different mechanisms, including increased nerve proliferation (Cai et al., 2007), improved nerve regeneration (Li et al., 2003), reduced inflammatory cytokine and neuronal apoptosis (Chen et al., 2001). These mechanisms are greatly associated with the pathophysiological process and clinical outcome in the acute and subacute phases. To date, only two studies demonstrated the mechanisms of angiogenesis by the expressions of VEGF and Flk at the acute stage of ischaemic brains (Cai et al., 2007; Cai and Liu, 2010). The present study showed that ischemia-induced Ang-1 expression was not enough for vascular remodeling, and that BYHWD treatment improved neurological behavior performance and reduced the infarct and atrophy volumes. Furthermore, it increased microvessel formation and the secretion of endogenous Ang-1 surrounding the ischemic tissue 
in the chronic stage. Our findings thus provide new insights into the possible regulatory mechanisms of BYHWD for neurorestorative therapy.

The Ang-1/Tie-2 receptor system, as one of the most potent angiogenic factors, was expressed during the later stages of stroke. Lin et al. (2000) demonstrated that Ang-1 mRNA slightly increased shortly after ischemia and found an eightfold increase $7-14 \mathrm{~d}$ after the insult, and that Tie-2 mRNA levels were observed to remain at approximately the fourfold level for up to two weeks. Zhang et al. (1999) reported an increase of Ang- 1 mRNA at $24 \mathrm{~h}$ postischemia, which persisted up to $28 \mathrm{~d}$. Our data also showed that Ang-1 proteins were up-regulated to a high level at the end of two weeks and decreased gradually until four weeks after focal cerebral ischemia in the vehicle-treated group. However, BYHWD treatment increased and maintained the expression of endogenous Ang-1 until three weeks. Moreover, the Ang-1/Tie-2 receptor system exerts its functions on vascular remodeling and neurorestorative processes at chronic stages, i.e., when the microvascular beds was stabilized by the remodeling and maturation of arterioles, capillaries and venules into a complex hierarchical network. Ang-1 promotes the survival and migration of endothelial cells and blood-vessel maturation and exerts a vessel-sealing effect (Suri et al., 1996; Thurston et al., 2000). It appears to have recruited precursors into the wall of developing vessels, in which the cells are transformed into pericytes and smooth muscle cells. Ang-1 and VEGF in combination induce a synergistic angiogenic effect, and promote the formation of mature neovessels without the side effects on BBB permeability after $7 \mathrm{~d}$ (Valable et al., 2005). The coexpression of Ang-1 with VEGF increases the structural integrity of the BBB and reduces atrophy volume at three weeks (Shen et al., 2011). Our data further showed that vascularization surrounding the ischemic tissue was formed and the atrophy volume was reduced in the chronic phages at four weeks after BYHWD treatment.

In addition to its angiogenic functions, Ang-1/ Tie-2 receptor system is also involved in neurogenesis, which along with angiogenesis might be directly linked by Ang-1/Tie-2. In our study, Ang-1 protein was concentrated not only in brain microvascular endothelial cells constructing the vascular structures, but also in the nerve tissue of the penumbra area, which may indirectly indicate that the Ang-1/ Tie-2 signal has a combinative role in the neurorestorative process. Reports also suggest that the expression of Ang-1 was found in neurons and neural stem cells, and that exogenous Ang-1 promotes the survival of neurons and neural progenitor cells after exposure to serum deprivation or hypoxia (Valable et al., 2003; Bai et al., 2009). Ang-1 also elicits neuronal differentiation and neurite outgrowth in mouse embryonic cortical and dorsal root ganglion cells (Kosacka et al., 2005; Bai et al., 2009). After cortical and striatal stroke, ischemia elicits an up-regulation of Ang-1 and the migration of the doublecortin (DCX)positive neuroblasts from the subventricular zone to the peri-infarct area (Yamashita et al., 2006; Shin et al., 2008). Moreover, neuroblasts have been found to be concentrated around blood vessels following stroke (Yamashita et al., 2006).

In conclusion, the results of the present study show that BYHWD modulates angiogenesis in the boundary ischemic region and increases the expressions of Ang-1, which may be the mechanism by which BYHWD ameliorates chronic brain injury after focal cerebral ischemia in rats. Taken together, the results suggest that BYHWD may improve collateral circulation and provide certain impacts for stroke patients through new blood vessel formation. Future research may elucidate the specific signaling pathways through which BYHWD increases angiogenesis.

\section{Compliance with ethics guidelines}

Jian SHEN, Yu ZHU, Hai YU, Zuo-xu FAN, Feng XIAO, Pan WU, Qi-hui ZHANG, Xiao-xing XIONG, Jian-wei PAN, and Ren-ya ZHAN declare that they have no conflict of interest. All institutional and national guidelines for the care and use of laboratory animals were followed.

\section{References}

Bai, Y., Cui, M., Meng, Z., et al., 2009. Ectopic expression of angiopoietin-1 promotes neuronal differentiation in neural progenitor cells through the Akt pathway. Biochem. Biophys. Res. Commun., 378(2):296-301. [doi:10.1016/ j.bbrc.2008.11.052]

Beck, H., Plate, K.H., 2009. Angiogenesis after cerebral ischemia. Acta Neuropathol., 117(5):481-496. [doi:10. 1007/s00401-009-0483-6] 
Bederson, J.B., Pitts, L.H., Tsuji, M., et al., 1986. Rat middle cerebral artery occlusion: evaluation of the model and development of a neurologic examination. Stroke, 17(3): 472-476. [doi:10.1161/01.STR.17.3.472]

Bramlett, H.M., Dietrich, W.D., 2004. Pathophysiology of cerebral ischemia and brain trauma: similarities and differences. J. Cereb. Blood Flow Metab., 24(2):133-150. [doi:10.1097/01.WCB.0000111614.19196.04]

Cai, G.X., Liu, B.Y., 2010. Buyang Huanwu decoction increases vascular endothelial growth factor expression and promotes angiogenesis in a rat model of local cerebral ischemia. Neural. Regen. Res., 5:1733-1738 (in Chinese).

Cai, G.X., Liu, B.Y., Liu, W., et al., 2007. Buyang Huanwu decoction can improve recovery of neurological function, reduce infarction volume, stimulate neural proliferation and modulate VEGF and Flk1 expressions in transient focal cerebral ischaemic rat brains. J. Ethnopharmacol., 113(2):292-299. [doi:10.1016/j.jep.2007.06.007]

Chen, J., Chopp, M., 2006. Neurorestorative treatment of stroke: cell and pharmacological approaches. NeuroRX, 3(4):466-473. [doi:10.1016/j.nurx.2006.07.007]

Chen, Y.S., Cheng, W.C., Yao, C.H., et al., 2001. Effects of Buyang Huanwu decoction on peripheral nerve regeneration using silicone rubber chambers. Am. J. Chin. Med., 29(03n04):423-432. [doi:10.1142/S0192415X0100 0447]

Fan, Y., Yang, G.Y., 2007. Therapeutic angiogenesis for brain ischemia: a brief review. J. Neuroimmune Pharmacol., 2(3):284-289. [doi:10.1007/s11481-007-9073-3]

Gu, L., Xiong, X., Wei, D., et al., 2013. T cells contribute to stroke-induced lymphopenia in rats. PLoS ONE, 8(3): e59602. [doi:10.1371/journal.pone.0059602]

Hao, C.Z., Wu, F., Shen, J., et al., 2012. Clinical efficacy and safety of Buyang Huanwu decoction for acute ischemic stroke: a systematic review and meta-analysis of 19 randomized controlled trials. Evid. Based Complement. Alternat. Med., 2012:630124. [doi:10.1155/2012/630124]

Kosacka, J., Figiel, M., Engele, J., et al., 2005. Angiopoietin-1 promotes neurite outgrowth from dorsal root ganglion cells positive for Tie-2 receptor. Cell Tissue Res., 320(1): 11-19. [doi:10.1007/s00441-004-1068-2]

Li, X.M., Bai, X.C., Qin, L.N., et al., 2003. Neuroprotective effects of Buyang Huanwu decoction on neuronal injury in hippocampus after transient forebrain ischemia in rats. Neurosci. Lett., 346(1-2):29-32. [doi:10.1016/S03043940(03)00522-6]

Lin, T.N., Wang, C.K., Cheung, W.M., et al., 2000. Induction of angiopoietin and Tie receptor mRNA expression after cerebral ischemia-reperfusion. J. Cereb. Blood Flow Metab., 20(2):387-395. [doi:10.1097/00004647-20000 2000-00021]

Liu, X.B., Chen, H., Chen, H.Q., et al., 2012. Angiopoietin-1 preconditioning enhances survival and functional recovery of mesenchymal stem cell transplantation. J. Zhejiang
Univ.-Sci. B (Biomed. \& Biotechnol.), 13(8):616-623. [doi:10.1631/jzus.B1201004]

Pang, L., Ye, W., Che, X.M., et al., 2001. Reduction of inflammatory response in the mouse brain with adenoviralmediated transforming growth factor- $\beta 1$ expression. Stroke, 32(2):544-552. [doi:10.1161/01.STR.32.2.544]

Shen, F., Walker, E.J., Jiang, L., et al., 2011. Coexpression of angiopoietin-1 with VEGF increases the structural integrity of the blood-brain barrier and reduces atrophy volume. J. Cereb. Blood Flow Metab., 31(12):2343-2351. [doi:10.1038/jcbfm.2011.97]

Shin, H.Y., Kim, J.H., Phi, J.H., et al., 2008. Endogenous neurogenesis and neovascularization in the neocortex of the rat after focal cerebral ischemia. J. Neurosci. Res., 86(2):356-367. [doi:10.1002/jnr.21494]

Suri, C., Jones, P.F., Patan, S., et al., 1996. Requisite role of angiopoietin-1, a ligand for the TIE2 receptor, during embryonic angiogenesis. Cell, 87(7):1171-1180. [doi:10. 1016/S0092-8674(00)81813-9]

Suri, C., McClain, J., Thurston, G., et al., 1998. Increased vascularization in mice overexpressing angiopoietin-1. Science, 282(5388):468-471. [doi:10.1126/science.282. 5388.468]

Thurston, G., Rudge, J.S., Ioffe, E., et al., 2000. Angiopoietin-1 protects the adult vasculature against plasma leakage. Nat. Med., 6(4):460-463. [doi:10.1038/74725]

Valable, S., Bellail, A., Lesné, S., et al., 2003. Angiopoietin-1induced phosphatidyl-inositol 3-kinase activation prevents neuronal apoptosis. FASEB J., 17(3):443-445. [doi:10. 1096/fj.02-0372fje]

Valable, S., Montaner, J., Bellail, A., et al., 2005. VEGF-induced BBB permeability is associated with an MMP-9 activity increase in cerebral ischemia: both effects decreased by Ang-1.J. Cereb. Blood Flow Metab., 25(11):1491-1504. [doi:10.1038/sj.jcbfm.9600148]

Wang, H.W., Liou, K.T., Wang, Y.H., et al., 2011. Deciphering the neuroprotective mechanisms of Bu-yang Huan-wu decoction by an integrative neurofunctional and genomic approach in ischemic stroke mice. J. Ethnopharmacol., 138(1):22-33. [doi:10.1016/j.jep.2011.06.033]

Wei, L., Erinjeri, J.P., Rovainen, C.M., et al., 2001. Collateral growth and angiogenesis around cortical stroke. Stroke, 32(9):2179-2184. [doi:10.1161/hs0901.094282]

Weidner, N., 2008. Chapter 14. Measuring intratumoral microvessel density. Methods Enzymol., 444:305-323. [doi:10.1016/S0076-6879(08)02814-0]

Weintraub, M.I., 2006. Thrombolysis (tissue plasminogen activator) in stroke: a medicolegal quagmire. Stroke, 37(7): 1917-1922. [doi:10.1161/01.STR.0000226651.04862.da]

Xiong, X.X., Gu, LJ., Zhang, H.F., et al., 2013. The protective effects of $\mathrm{T}$ cell deficiency against brain injury are ischemic model-dependent in rats. Neurochem. Int., 62(3): 265-270. [doi:10.1016/j.neuint.2012.11.016]

Yamashita, T., Ninomiya, M., Hernandez-Acosta, P., et al., 
2006. Subventricular zone-derived neuroblasts migrate and differentiate into mature neurons in the post-stroke adult striatum. J. Neurosci., 26(24):6627-6636. [doi:10. 1523/JNEUROSCI.0149-06.2006]

Zhang, Z.G., Chopp, M., Lu, D., et al., 1999. Receptor tyrosine kinase tie 1 mRNA is upregulated on cerebral microvessels after embolic middle cerebral artery occlusion in rat.
Brain Res., 847(2):338-342. [doi:10.1016/S0006-8993 (99)02013-2]

Zhao, L.D., Wang, J.H., Jin, G.R., et al., 2012. Neuroprotective effect of Buyang Huanwu decoction against focal cerebral ischemia/reperfusion injury in rats-time window and mechanism. J. Ethnopharmacol., 140(2):339-344. [doi: 10.1016/j.jep.2012.01.026]

\section{中文碳要:}

本文题目: 补阳还五汤增加脑缺血后血管生成素-1 的表达及促进血管生成和神经功能改善

Buyang Huanwu decoction increases angiopoietin-1 expression and promotes angiogenesis and functional outcome after focal cerebral ischemia

研究目的：补阳还五汤对脑缺血后缺血区血管生成的影响及其机制。

创新要点: 研究结果表明补阳还五汤可能通过增加血管生成素-1 的表达, 促进缺血区血管新生, 从而促 进脑梗死后神经功能恢复。

研究方法: 通过神经功能评分、脑梗死及脑萎缩体积评估神经功能恢复情况; 通过免疫组化及免疫印迹 等方法检测血管密度及血管生成因子的表达情况。

重要结论: 补阳还五汤增加脑缺血后血管生成素-1 的表达, 能够促进脑梗死缺血区血管生成。

关键词组: 补阳还五汤; 血管生成素-1；血管生成；慢性脑损伤；神经修复 